\title{
Austenite-ferrite interface crystallography dependence of sigma phase precipitation using the five-parameter characterization approach
}

\author{
N. Haghdadi ${ }^{1}$, D. Abou-Ras ${ }^{2}$, P. Cizek$^{1}$, P.D. Hodgson ${ }^{1}$, A. D. Rollett ${ }^{3},{ }^{* H}$. Beladi ${ }^{1}$ \\ ${ }^{1}$ Institute for Frontier Materials, Deakin University, Waurn Ponds, VIC 3216, Australia \\ ${ }^{2}$ Helmholtz-Zentrum Berlin für Materialien und Energie GmbH, Hahn-Meitner-Platz 1, 14109 Berlin, Germany \\ ${ }^{3}$ Department of Materials Science and Engineering, Carnegie Mellon University, Pittsburgh, PA 15213-3890, USA \\ * Corresponding Author: hossein.beladi@deakin.edu.au
}

\begin{abstract}
In the current study, the propensity of austenite-ferrite interface to sigma phase precipitation was investigated in a duplex stainless steel. The crystallographic characteristics of the interphase interfaces significantly affected the tendency to sigma phase precipitation. The lattice misorientation was found to be an insufficient criterion to fully define the interface character and ultimately the propensity of austenite-ferrite interface to sigma phase precipitation. The interphase interfaces with a rational orientation relationship, considering all five crystallographic parameters, generally exhibited the lowest sigma precipitation propensity. This could be attributed to the high sigma precipitation nucleation barrier energy and low diffusion rate at (semi-)coherent interphases.
\end{abstract}

Keywords: duplex stainless steel; sigma precipitation; austenite-ferrite interface; orientation relationship

\section{Introduction}

Duplex stainless steels are recently seeing increased use because of their attractive combination of strength and toughness as well as high corrosion resistance [1]. Optimization of these steels is, however, challenging because of their complex precipitation and transformation behavior. The toughness and corrosion resistance of duplex stainless steels is adversely affected by the formation of carbides, nitrides, and intermetallic phases [2]. In particular, sigma phase $(\sigma)$ is readily precipitated, which is known to have detrimental impact on the properties of stainless steel, especially ductility [3]. It is, therefore, critically important to understand the parameters affecting sigma precipitation to limit its formation and ultimately enhance the material performance. The effect of steel chemistry and heat treatment conditions (temperature and time) on the kinetics of sigma precipitation has been extensively 
studied [4-6]. Sigma preferentially nucleates on austenite-ferrite interfaces and grows into the ferrite. The ferrite to austenite transformation is accompanied by the alloying elements partitioning. Therefore, the compositional variation at the interface of ferrite/austenite is inevitable, though the extent of the variation depends on the cooling rate. Despite significant body of work on sigma precipitation, little is known about the influence of the interphase boundary character on the precipitation of sigma, which this paper aims to remedy.

Honeycombe [7] was among the first to suggest that the interfaces with low mobility might be preferential precipitation nucleation sites via the ledge mechanism. In duplex stainless steels, austenite-ferrite interfaces with rational orientation relationship/s (e.g., Kurdjumov-Sachs) exhibit the minimum energy with low mobility [7]. Therefore, it is reasonable to hypothesize that such rational interfaces are the preferred sites for precipitation in dual-phase steels. However, a recent study on low-carbon steels [8] revealed that VC interphase precipitation in both sheet-like and random dispersions was promoted in the austenite-ferrite interfaces that deviate from the Kurdjumov-Sachs (K-S) orientation relationship (OR). This is consistent with another report on welded duplex stainless steels [9], where sigma phase mostly precipitated on interfaces far from the K-S orientation relationship. This was attributed to the increase in interface energy with increasing deviation from the exact orientation relationship.

The previous studies characterized the austenite-ferrite interface using only the misorientation angle/axis (i.e. lattice misorientation), which contains three parameters $[8,9]$. However, five independent crystallographic parameters are required to fully characterize interphases and boundaries in polycrystalline materials [5]. These include the interface plane orientation (or, inclination) in addition to the lattice misorientation, which cannot be directly extracted from orientation maps (as is the case for misorientation). In other words, the previous studies did not take into account the role of interphase plane/s character on the precipitation in two-phase microstructures. Studies on single-phase aluminum alloys revealed that the grain boundary plane character plays a significant role in precipitation and that the lattice misorientation is not a sufficient criterion [12]. Therefore, the current study aims to study the propensity of austenite-ferrite interfaces for sigma precipitation in a duplex stainless steel using all five crystallographic parameters. This provides a new insight into the sigma precipitation in duplex stainless steels, which has both fundamental and applied significance in light of the growing importance of this class of steels.

\section{Experimental}


The material used was a commercial 2205 duplex stainless steel with a chemical composition of $0.036 \mathrm{C}, 0.321 \mathrm{Si}, 1.82 \mathrm{Mn}, 0.013 \mathrm{P}, 23.2 \mathrm{Cr}, 2.90 \mathrm{Mo}, 5.6 \mathrm{Ni}, 0.153 \mathrm{Cu}, 0.245 \mathrm{~N}$ (in wt. \%) and remainder $\mathrm{Fe}$. The initial material was received as a hot-rolled slab. Two specimens were reheated to $1370{ }^{\circ} \mathrm{C}$ and held isothermally for $40 \mathrm{~min}$ in a muffle furnace in an argon atmosphere. They were then furnace cooled from $1370{ }^{\circ} \mathrm{C}$ to temperatures of $970{ }^{\circ} \mathrm{C}$ and $900{ }^{\circ} \mathrm{C}$ followed by water quenching. The average cooling rate was estimated to be about $0.002{ }^{\circ} \mathrm{C} / \mathrm{s}$. This reduces the compositional variation at the ferrite/austenite interfaces. The electron backscattered diffraction (EBSD) technique was employed to characterize the microstructure. Routine EBSD sample preparation, as described elsewhere [13], was employed. EBSD data acquisition was carried out using an FEI Quanta 3D FEG SEM/FIB instrument, equipped with a TexSEM Laboratories (TSL OIM) EBSD attachment, operated at $20 \mathrm{kV}$. The EBSD post-processing analysis was conducted using the TSL software. More than 500 different austenite-ferrite interfaces were analyzed to evaluate the propensity of sigma precipitation with respect to the austenite-ferrite interface characteristics. The interfaces were classified in terms of all five crystallographic interface parameters, namely misorientation angle, misorientation axis and plane orientation using the TSL software in conjunction with a trace analysis approach.

\section{Results and discussion}

The heat treatment of the material at $1370{ }^{\circ} \mathrm{C}$ resulted in a coarse $\delta$-ferrite (hereafter called ferrite) grain structure with an average size of $360 \pm 35 \mu \mathrm{m}$, which progressively transformed to austenite during furnace cooling to $970{ }^{\circ} \mathrm{C}$ (Figs. 1a,b). At this temperature, the volume fraction of austenite was $50 \pm 4 \%$ having an average grain size of $100 \pm 12 \mu \mathrm{m}$. It should be noted that no new phases (as particles) were apparent in the microstructure. Further cooling led to a slight increment in the volume fraction of austenite $(53 \pm 4 \%)$ but, most importantly, new phases appeared in the microstructure. Sigma phase was the dominant precipitate formed (Figs. 1c,d). In addition to sigma, $\chi$ phase and chromium nitride also precipitated during cooling from $970{ }^{\circ} \mathrm{C}$ to $900{ }^{\circ} \mathrm{C}$. These are the most commonly observed secondary phases precipitated in duplex stainless steels at temperatures below $\sim 950{ }^{\circ} \mathrm{C}$ [1]. The sigma phase may form through different mechanisms. It can be directly formed on the austenite-ferrite interface or as a result of a eutectoid phase transformation (ferrite $\rightarrow$ austenite + sigma). The gradual transformation of $\chi$ and $\mathrm{Cr}_{2} \mathrm{~N}$ to sigma phase may also occur during further aging [14]. The latter transformations $\left(\mathrm{Cr}_{2} \mathrm{~N}\right.$ to sigma, in particular), however, need a prolonged annealing time [14]. 
Small isolated austenite islands were frequently observed in the ferrite interior in the vicinity of the sigma particles. This suggests that the ferrite locally underwent eutectoid phase transformation to sigma and austenite [15]. As a result, the volume fraction of ferrite decreased to $23 \pm 5 \%$ at $900{ }^{\circ} \mathrm{C}$. Sigma particles mostly appeared on the austenite ferrite interfaces and were elongated into the ferrite (Figs. 1c,d). Therefore, it would be expected that these precipitates retard the migration of austenite-ferrite interfaces through the pinning effect. Interestingly, sigma precipitates were not observed on all ferrite-austenite interfaces and some of them were precipitation free. This suggests that the propensity of sigma precipitation is linked to the interface crystallographic characteristics.

In the current study, the crystallography of more than 500 austenite-ferrite interfaces was analyzed using EBSD data. The misorientation angle and axis distributions were plotted in Figure 2 for austenite-ferrite interfaces with different precipitation propensities. The misorientation angles of all interfaces exhibited a broad distribution in a range of 10 to $60^{\circ}$, having a peak at $\sim 44^{\circ}$ (Fig. 2a). The interfaces were classified into two categories based on the occurrence of sigma precipitation: i) interfaces containing sigma precipitates and ii) sigma-free interfaces (Figs. 2b,c). The misorientation angle distribution for precipitate-free interfaces was significantly different from that associated with precipitates. It has a narrow distribution with a distinct peak in a range of $40-50^{\circ}$ (Fig. 2b). By contrast, the interfaces with precipitates showed a wide misorientation angle distribution with a shallow peak in a range of 35 to $45^{\circ}$ (Fig. 2c). In general, the interfaces with misorientation angles between 40 and $50^{\circ}$ revealed a lower tendency to sigma precipitation compared to the rest. Interestingly, this misorientation angle range overlaps with that expected for the well-known OR models (e.g., K-S, Nishiyama-Wasserman (N-W), Greninger-Troiano (G-T) and Pitsch (P) [16]) that apply to the BCC to FCC transformation. The K-S and N-W ORs, which differ by only $5.26^{\circ}$, are the most generally used models for this phase transformation. The other proposed models (e.g., Pitsch and G-T) lie between K-S and N-W ORs. The misorientation angle/axis pairs for K-S and N-W ORs are 42.85\% $/<0.9680 .1780 .178>$ and $45.98^{\circ} /<0.9760 .0830 .201>$, respectively. The lattice plane/direction corresponding to these ORs are $\{110\}<111>_{\mathrm{F}} / /$ $\{111\}<011>_{\mathrm{A}}$ and $\{110\}<100>_{\mathrm{F}} / /\{111\}<011>_{\mathrm{A}}$, respectively [17].

To further investigate the lattice misorientation relationship between austenite and ferrite for a given interface, one should also consider the rotation axis in addition to the misorientation angle. Figure 3 plots the misorientation axes associated with different misorientation angles between $42^{\circ}$ and $46^{\circ}$ for the interfaces containing sigma precipitates. In the current study, misorientations deviated up to 10 degrees from ideal KS/NW ORs were 
considered as rational interfaces. It is clear that the misorientation axes of interfaces with precipitates deviate significantly from those associated with K-S and N-W ORs (i.e. $<0.9680 .1780 .178>$ and $<0.9760 .0830 .201>$, respectively). It is interesting to note that sigma precipitation was also observed in some interfaces having their misorientation angle/axis close to K-S and N-W ORs. However, it is not clear yet whether these interfaces also fulfilled the plane parallelism requirement associated with $\mathrm{K}-\mathrm{S}$ and N-W ORs (i.e., $\left.\{110\}_{\mathrm{F}} / /\{111\}_{\mathrm{A}}\right)$.

The austenite and ferrite planes associated with different interfaces were estimated using the trace analysis approach. It should be noted that this approach is a close approximation, as the determination of the exact interface planes would require a threedimensional examination [18]. Here, oriented stereographic projections were constructed based on overlaying the austenite and ferrite pole figures, using the Euler angles of the phases on either side of a given austenite-ferrite interface. The matching planes were found by selecting the plane poles in the projection that were situated on the locus of possible interface plane normals (i.e. on the great circle perpendicular to the interface trace) and were closest to coincidence. As the sample was cooled slowly in the furnace (i.e., $\sim 0.002{ }^{\circ} \mathrm{C} / \mathrm{s}$ on average), it can be assumed that the interfaces formed through the ferrite to austenite phase transformation have relatively low energy arrangements (i.e., low index plane/s). Therefore, we only plotted pole figures for six low-index planes, namely $\{001\},\{011\},\{111\},\{012\}$, $\{112\}$ and $\{122\}$.

The trace analysis was performed on 100 interfaces consisting of 50 precipitated and 50 precipitate-free interfaces, all having their lattice misorientations close to K-S or N-W ORs. In the case of precipitated interfaces, the planes mostly deviated from those expected for both ideal K-S and N-W ORs ( $\sim 86 \%$, Table 1$)$. Figure $4 \mathrm{~b}$ represents an example of such a precipitated interface showing (012) austenite and (100) ferrite planes on either side of the interface. By contrast, most sigma-free interfaces $(\sim 60 \%$, Table 1) terminated on (111) austenite and (110) ferrite planes (e.g., Fig. 4a), corresponding to the ideal K-S and N-W ORs. In other words, the interfaces that fulfill all five crystallographic parameters defined by the above ORs displayed the lowest tendency towards precipitation (e.g., Fig. 4b). It should be noted that some irrational interfaces were also free of sigma precipitates, which could be a consequence of local composition inhomogeneity in the microstructure. This is evident in Fig. 1c where some regions appeared to locally have a stronger tendency to form precipitates than others. 
Honeycombe [7] initially suggested that interfacial precipitates form through the ledge mechanism and nucleate only on coherent interfaces due to their low mobility. According to this model, K-S interfaces are preferential sites for precipitation. However, the recent work, which characterized the interfaces based on only lattice misorientation criterion, demonstrated that the interfaces close to K-S ORs have the least frequency of sigma precipitation [9]. This is consistent with the current observations. Nevertheless, the present results reveal that the lattice misorientation is not a sufficient criterion to fully characterize the interface character and ultimately its precipitation propensity. The five-parameter analysis presented here for the first time demonstrates that the interface plane character plays a significant role in the precipitation tendency for interfaces with a lattice misorientation close to the K-S or N-W OR. This is consistent with the interface minimum energy estimated for the (110) ferrite and (111) austenite planes in the ideal K-S OR using the first nearest neighbor bond model [19]. Any deviation from these close-packed planes leads to an increase in the interface energy, which should decrease the activation energy barrier for sigma precipitation and also enhance the diffusivity of alloying elements in the vicinity of an interface [20].

Note that $\sim 63 \%$ of interfaces characterized by K-S or N-W OR, based on the lattice misorientation, did not fulfill the plane constraints associated with these ORs. This suggests that the austenite, to some extent, follows $\mathrm{K}-\mathrm{S}$ and $\mathrm{N}-\mathrm{W}$ ORs with the parent ferrite at an initial stage of transformation to minimize the activation energy of the nucleation [21]. In this circumstance, the austenite nucleated at the ferrite-ferrite grain boundaries maintains its lattice misorientation during the growth stage, at least with a ferrite grain at one side of the boundary. Thus, the lattice misorientation is dictated by the nucleation and remains unchanged while the austenite grows in a strain-free ferrite grain. The interphase plane may locally change during the growth, as it is controlled by the diffusional partitioning of alloying elements rather than the crystallographic constraints. Hence, the interface planes may become irrational and deviate from the ideal crystallographic planes (i.e. (111)A // (110)F).

\section{Conclusion}

The tendency of austenite-ferrite interfaces to precipitate sigma phase has been studied in a duplex stainless steel in the present investigation. The crystallography of the austenite-ferrite interface was found to affect the propensity to sigma phase precipitation. Considering only the lattice misorientation was found to be insufficient for understanding the effect of the interphase character on sigma phase precipitation. Austenite-ferrite interfaces with a rational 
orientation relationship, considering all five crystallographic parameters, exhibited the lowest tendency for precipitation. This is attributed to the high sigma precipitation nucleation barrier energy and low diffusion rate at (semi-)coherent interphases.

\section{Acknowledgements}

The present work was carried out with the support of the Deakin Advanced Characterisation Facility. Financial support provided by the Australian Research Council is gratefully acknowledged.

\section{References}

[1] R.N. Gunn, Duplex Stainless Steels: Microstructure, Properties and Applications, Woodhead Publishing, Cambridge, 1997.

[2] M. Pohl, O. Storz, T. Glogowski, Mater. Charact. 58 (2007) 65-71.

[3] G. Fargas, M. Anglada, A. Mateo, J. Mater. Process. Technol. 209 (2009) 1770-1782.

[4] S. Henrik, R. Sandström. Mater. Sci. Eng. A 444 (2007) 271-276.

[5] J.J. Eckenrod, K.E. Pinnow In: New Developments in Stainless Steel Technology; Metals Park, American Society for Metals, (1985) 77-87.

[6] Z. Wei, J. Laizhu, H. Jincheng, S. Hongmei, Mater. Charact. 60 (2009) 50-55.

[7] R.W.K. Honeycombe, R. F. Mehl, Metall. Mater. Trans. A 7 (1976) 915-936.

[8] Y.J. Zhang, G. Miyamoto, K. Shinbo, T. Furuhara, Scripta Mater. 69 (2013) 17-20.

[9] Y.S. Sato, H. Kokawa, Scripta Mater. 40 (1999) 659-663.

[10] G.S. Rohrer, D.M. Saylor, B.E. Dasher, B.L. Adams, A.D. Rollett, P. Wynblatt, Z. Metallkd. 95 (2004) 197-214.

[11] H. Beladi, G.S. Rohrer, Acta Mater. 61 (2013) 1404-1412.

[12] D. Vaughan, Acta Metal. 18 (1970) 183-187.

[13] N. Haghdadi, P. Cizek, H. Beladi, P. D. Hodgson, Acta Mater 126 (2017) 44-57.

[14] K.W. Chan, S.C. Tjong, Materials 7 (2014) 5268-5304.

[15] D.C. Dos Santos, R. Magnabosco, Metall. Mater. Trans. A 47 (2016) 1554-1565.

[16] Y. He, S. Godet, J.J. Jonas, J. Appl. Crystallogr. 39 (2006) 72-81.

[17] G. Nolze, Cryst. Res. Technol. 41 (2006) 72-77.

[18] X. Liu, N.T. Nuhfer, A.D. Rollett, S. Sinha, S-B. Lee, J.S. Carpenter, J. E. LeDonne, A. Darbal, K. Barmak, Acta Mater. 64 (2014) 333-344.

[19] J.K. Mackenzie, A.J.W. Moore, J.F. Nicholas, J. Phys. Chem. Solids 23 (1962) 185-196.

[20] R. Monzen, H. Shigehara, K. Kita, J. Mater. Sci. 23 (2000) 5839-5843. 
[21] H.K.D.H. Bhadeshia, Bainite in steels, Inst. of Metals, 1992. 